\title{
Reação Vascular Crônica Secundária à Sobreposição de Stents Farmacológicos
}

\author{
Ver artigo relacionado \\ na página 228
}

\author{
George Dangas ${ }^{1}$, Jiro Aoki ${ }^{1}$
}

C rescentes evidências sustentam os benefícios clínicos dos stents farmacológicos (DES) em um amplo espectro de pacientes e tipos de lesões. A segurança e a eficácia da sobreposição dos DES, em evolução de médio prazo, têm sido relatadas em estudos randomizados e registros que comparam alguns DES versus stents não-farmacológicos ${ }^{1-3}$. Entretanto, recentemente, a atenção tem sido voltada para a segurança em longo prazo dos DES, o chamado "problema da trombose tardia". A endotelização retardada do stent, assim como a resposta biológica aos polímeros duráveis e antiproliferativos residuais que permanecem no polímero, podem acentuar a trombogenicidade do DES. Em particular, a sobreposição no implante do DES é considerada um importante fator de risco para a trombose tardia do stent, porque a eluição de concentrações mais altas de fármacos do segmento sobreposto pode deteriorar o processo de cicatrização do vaso. Além disso, a fratura do stent também pode ocorrer, especialmente se a sobreposição estiver localizada em um segmento de vaso tortuoso e submetido a forças cardíacas de flexão.

De fato, segmentos onde ocorre a sobreposição dos DES estiveram associados à endotelização retardada e inflamação persistente, quando comparados aos stents não-farmacológicos, em estudo patológico experimental ${ }^{4}$. Isso sugere que a toxicidade arterial local pode potencialmente desenvolver-se quando o tecido adjacente é exposto a níveis inadequados de fármacos e/ou polímeros. Chama a atenção que, nesse estudo experimental, os achados patológicos dos stents com sirolimus (SES) e com paclitaxel (PES) foram diferentes. A sobreposição dos segmentos dos PES induziu maior deposição de heterófilos/eosinófilos luminais e de fibrina do que os SES; a infiltração de células gigantes ao redor das hastes do stent, entretanto, foi mais freqüente nos últimos.

Esses diferentes achados patológicos não se refletiram em estudos clínicos. Um estudo unicêntrico comparou as evoluções clínicas de 55 pacientes com sobreposição de SES e 59 pacientes com sobreposição de
PES $^{3}$. Em 30 dias e 6 meses de acompanhamento, a evolução clínica foi similar. Entretanto, há escassez de dados a respeito da comparação do crescimento neointimal nos segmentos de sobreposição dos SES e PES.

Neste número da Revista Brasileira de Cardiologia Invasiva, Lasave et al. ${ }^{5}$ mostram uma comparação dos segmentos com sobreposição de PES e SES, usando dados de ultra-sonografia intravascular (IVUS) do estudo randomizado REALITY. As mudanças volumétricas encontradas dentro e fora do stent nos segmentos de sobreposição (volume neointimal, porcentual de obstrução neointimal e razão de expansão do vaso) foram comparáveis entre SES e PES. Nesse estudo, o porcentual de obstrução intra-stent foi $18,15 \%$ versus $26,73 \%$ ( $p>0,1)$. Em geral, o efeito da inibição neointimal do SES é maior do que do PES. No Randomized Comparison of Sirolimus and Paclitaxel Drug-Eluting Stents for Long Lesions in the Left Anterior Descending Artery, o porcentual de obstrução intra-stent foi $7,4 \%$ versus $15,4 \% \quad(p<0,001)^{6}$. Chama a atenção que os valores do porcentual de obstrução intra-stent no estudo atual foram maiores do que no estudo prévio. A maior eluição dos fármacos antiproliferativos no segmento de sobreposição poderia ser ineficiente e a toxicidade arterial conseqüente sobrepujaria o efeito antiproliferativo da dose elevada. Para se esclarecer este achado, seria interessante comparar o crescimento neointimal entre os segmentos de sobreposição com aqueles sem sobreposição de hastes entre os SES e PES.

O remodelamento do vaso após o implante do DES é também um tópico interessante. O aumento da placa atrás do stent foi excessivo após o implante de PES de liberação moderada comparado ao de liberação lenta e esse fenômeno foi explicado presumidamente pela eluição elevada do paclitaxel, na fase inicial de liberação do primeiro modelo. O implante de stents com sobreposição mostra-se útil como um modelo internamente controlado, que permite, teoricamente, comparar a reação crônica do vaso às altas e baixas

1. Columbia University Medical Center, Cardiovascular Research Foundation, New York, USA.

Correspondência: George Dangas, MD, PhD. Columbia University Medical Center, New York, NY - 111 East 59 th Street, $11^{\text {th }}$ Floor - New York, NY, USA 10022 - Tel: 2128419151 - Fax: 2128519346 - E-mail: gdangas@crf.org

Recebido em: 08/07/2007 - Aceito em: 27/07/2007 
doses locais dos antiproliferativos. Conseqüentemente, pode-se suspeitar que o segmento com a sobreposição esteja associado a maior remodelamento positivo do que o segmento sem sobreposição de hastes. Entretanto, o oposto foi evidente neste estudo, pois a razão de expansão do vaso (segmento de sobreposição/vaso fora do segmento de sobreposição) foi menor do que um, em ambos os grupos tratados com SES e PES.

Um ponto interessante que necessita de esclarecimento, quando segmentos sobrepostos de stent são avaliados, é a definição de vaso "fora do segmento de sobreposição". Isso pode incluir tanto os segmentos de vasos recobertos por hastes sem sobreposição (em um ou outro sentido), além das bordas do stent e do vaso de referência fora da área tratada. Todas as investigações relevantes devem ser muito precisas em relação às definições utilizadas nas análises executadas. Acreditamos que a comparação entre o segmento de sobreposição e o sem sobreposição dos SES e PES é útil para que seja compreendida a relação entre as mudanças volumétricas do vaso e a eluição elevada dos antiproliferativos.

Recentemente, a tecnologia de imagem coronária tem feito um progresso rápido. Estudos adicionais usando métodos invasivos de imagem, como a angioscopia, a tomografia de coerência óptica e a histologia virtual, são necessários para investigar os segmentos de so- breposição do DES e as diferentes reações crônicas do vaso, entre os SES e PES, em estudos clínicos in-vivo.

\section{REFERÊNCIAS BIBLIOGRÁFICAS}

1. Kereiakes DJ, Wang H, Popma JJ, Kuntz RE, Donohoe DJ, Schofer J, et al. Periprocedural and late consequences of overlapping Cypher sirolimus-eluting stents: pooled analysis of five clinical trials. J Am Coll Cardiol. 2006;48(1):21-31.

2. Stone GW, Ellis SG, Cannon L, Mann JT, Greenberg JD, Spriggs D, et al. Comparison of a polymer-based paclitaxeleluting stent with a bare metal stent in patients with complex coronary artery disease: a randomized controlled trial. JAMA. 2005;294(10):1215-23.

3. Chu WW, Kuchulakanti PK, Torguson R, Wang B, Clavijo LC, Suddath WO, et al. Comparison of clinical outcomes of overlapping sirolimus- versus paclitaxel-eluting stents in patients undergoing percutaneous coronary intervention. Am J Cardiol. 2006;98(12):1563-6.

4. Finn AV, Kolodgie FD, Harnek J, Guerrero LJ, Acampado E, Tefera K, et al. Differential response of delayed healing and persistent inflammation at sites of overlapping sirolimus- or paclitaxel-eluting stents. Circulation. 2005;112(2):270-8.

5. Lasave L, Costa Jr JR, Abizaid A, Sanchez A, Feres F, Costa $R$, et al. Análise comparativa do segmento de "ovelapping" dos stents liberadores de sirolimus e paclitaxel. Rev Bras Cardiol Invas. 2007;15(3):228-233.

6. Petronio AS, De Carlo M, Branchitta G, Papini B, Ciabatti N, Gistri R, et al. Randomized comparison of sirolimus and paclitaxel drug-eluting stents for long lesions in the left anterior descending artery: an intravascular ultrasound study. J Am Coll Cardiol. 2007;49(5):539-46. 